\title{
Simulation Pedestrian Flow Under Vertical Bottleneck Constraints: How Stair Configuration Affects Efficiency
}

\author{
Jianyu Wang ${ }^{1,2} \cdot$ Mei-Cheng Yang ${ }^{3} \cdot$ Majid Sarvi $^{2} \cdot$ Abdullah Alhawsawi $^{2}$. \\ Peng Lin ${ }^{4}$. Jian $\mathrm{Ma}^{\star 1}$ \\ ${ }^{1}$ School of Transportation and Logistics, National Engineering Laboratory of Integrated \\ Transportation Big Data Application Technology, Southwest Jiaotong University, Chengdu, \\ China, \\ E-mail: jianyuwang@my.swjtu.edu.cn, majian@swjtu.edu.cn, *corresponding author. \\ 2 Transport Engineering Group, Department of Infrastructure Engineering,The University of \\ Melbourne, Australia, \\ E-mail: jianyuw@student.unimelb.edu.au, majid.sarvi@unimelb.edu.au, \\ aalhawsawi@student.unimelb.edu.au \\ ${ }^{3}$ China Railway Eryuan Engineering Group Co.Ltd, Chengdu, China, \\ E-mail: yangmc727@163.com \\ ${ }^{4}$ Department of Fire Safety Engineering, Southwest Jiaotong University, Chengdu China, \\ E-mail: drag76@163.com
}

Received: 15 August 2021 / Last revision received: 15 November 2021 / Accepted: 22 November 2021 DOI: $10.17815 / \mathrm{CD} .2021 .126$

\begin{abstract}
As a result of the geometric constraints of the stairs, pedestrian movement features on stairways are different from level ambulation. Therefore, it is necessary for us to consider the stairs' three-dimensional geometry when we try to explore how the stair configuration affects pedestrian movement efficiency. Based on the pedestrians fundamental features from a previous single-file pedestrian movement experiment on stairs, we investigated pedestrian flow under various stair configurations with an improved agentbased model. Our simulated results indicated that both stair inclination and tread depth are sensitive parameters which affects pedestrian dynamic on stairs. Generally speaking, pedestrian flow decreased with the increasing slope of stairs. When the stairs slope increased 5, 10, 15 and 20 degree, the evacuation efficiency dropped 5.8\%, 12.8\%, 19.3\% and $28 . \%$. Besides, the effect of the tread depth on pedestrian total evacuation time is not changed monotonously. Scenarios with $0.3 \mathrm{~m}$ tread depth size presented the optimal evacuation efficiency. Other scenarios differed from $3 \%$ to $27.9 \%$ in terms of the total evacuation time.
\end{abstract}


Keywords Pedestrian dynamic · stairs movement · ascent and descent movement $\cdot$ stair configuration

\section{Introduction}

Urbanization promotes gradually in the past few decades, more and more complex structures such as high-rise buildings and multi-story underground buildings are built to relieve the increasing pressure on traffic congestion and inefficient use of space [1,2]. As a vertical channel connecting different building levels, stairs are most of the time the only means of egress in high-rise buildings, subway stations, shopping malls, cruise ships and other building facilities [3]. Walking on stairs is a very common and vital activity in our daily life $[4,5]$.

Researchers found that pedestrian flow rate on stairs is lower than that on the horizontal movement because congestion often occurs in front of the stairs. Such delays must be factored into evacuation plans, in that the stairwell usually becomes the only evacuation route under emergency conditions. Therefore, plenty of surveys, experiments and evacuation drills have been performed and pedestrian walking speed on stairway has become researchers topic in the past few decades [6-11]. Besides, in a recent study [12], we have confirmed the flow decreases with the increasing slope of stairs, and found the factors, i.e., tread depth $(T)$ and riser height $(R)$ of stair-step have a significant effect on pedestrian dynamic on stairs. These findings inspired us to consider how to quantify the effect of stair slope and tread depth on pedestrian movement.

Therefore, this paper aims to investigate how stair configuration affects pedestrian movement efficiency. This question will be discussed based on a series of pedestrian simulation works.

\section{Methodology}

The simulation model employed in this work is developed at The University of Melbourne. This model consists of two layers. At the operation level, the simulated agents' motion features were described by the social force model, and the parameters and rules were calibrated and optimized by [13] and [14]. At the tactical level, the route choice and path-finding of simulated agents are generated by using a discrete-choice model [15]. At each time step, the motion of each simulated agent is determined by the overall affection of the driven force, the interaction force between pedestrians and the repulsive force between pedestrians and obstacles.

In order to show the effect of the vertical bottleneck constraints on pedestrian movement efficiency, we considered different stair configurations for pedestrian ascending and descending movement. By comparing the common types of stairs in our daily life, academic publications and reports in the past few decades, and referring to the relevant building designs [12], we examined the stair slopes (see Fig. $1, \theta=20^{\circ}, 25^{\circ}, 30^{\circ}, 35^{\circ}$ and $40^{\circ}$ ) with three tread depth size (denoted as $T$, with $T=0.25,0.3$ and $0.35 \mathrm{~m}$ ). Therefore, in 
total, 30 scenarios were simulated. In each scenario, 150 agents are used (agents were initially distributed in the yellow area in Fig. 2(a) and 2(b)), which looked as a medium crowd size condition. Each scenario calculation was repeated 50 times.

The agent radius and agent mass we used in the simulations were referred to the empirical data set of individuals [16]. The velocity on the plane for each individual was set to $1.2 \mathrm{~m} / \mathrm{s}$. The simulated agents velocity on stairs was set according to the stair configurations and estimated by a linear regression model [12]. Tab. 1 provides the details of the pedestrian fundamental parameters, including the free movement ascending speed, free movement descending speed and free movement headway distance for each specific simulation scenario.

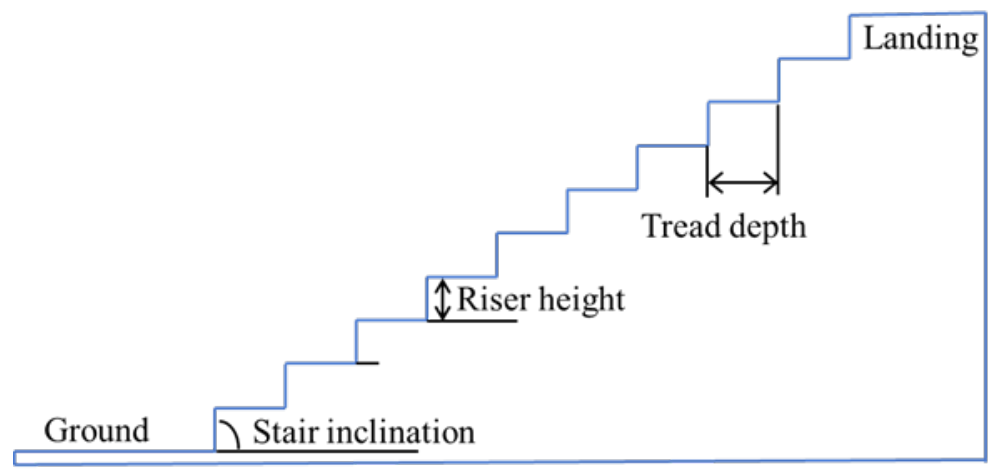

Figure 1 Schematic illustration of the stairs.

\section{Results}

Due to the accurate estimates of the total evacuation times (TET) has become a major concern related to the pedestrian capacity of crowded spaces [17-19], we averaged the total evacuation time for all the tested scenarios over 50 repetitions, as shown in Fig. 3. The dark color in the contour map of the combination effect of stair inclinations $\theta$ and tread depth $T$ represents the shorter total evacuation time, and on the contrary, the bright color represents a longer total evacuation time.

According to the data in Fig. 3, one immediate observation is that for all the tested scenarios, higher stair inclination shows a longer total evacuation time for simulated agents. This feature is reasonable in that with the increase of stair inclination, the influence of gravity on pedestrian movement increases gradually. Besides, the effect of the tread depth on TET is not changed monotonously. Under the same stair inclination conditions, scenarios with $T=0.3 \mathrm{~m}$ display the fastest $T$, then followed by $T=0.25 \mathrm{~m}$ series scenarios and the $\mathrm{T}=0.35 \mathrm{~m}$ series scenarios both for ascending and descending movement.

To quantitatively analyze the effect of the tread depth and stair inclination on pedestrian flow on stairs, we analyzed the data distribution in Fig. 3 horizontally and vertically with ANOVA statistical analysis method, respectively. Results are shown in Fig. 4 and Fig. 5. These two figures visualized the values of evacuation time and its average for each 

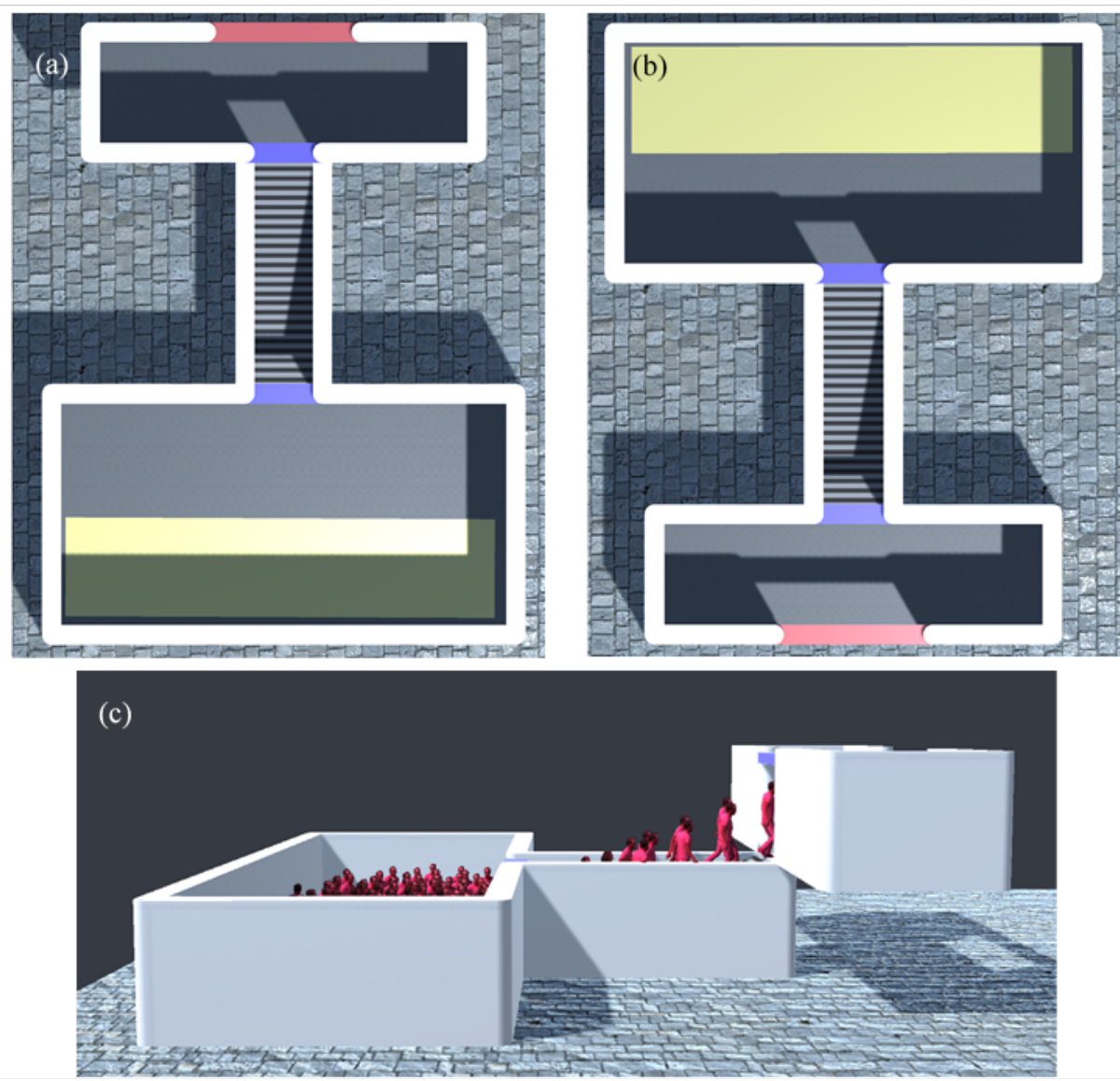

Figure 2 Snapshots of the pedestrian simulation: (a) for ascending, (b) for descending, (c) Still-image of the ascending simulation.

scenario associated with 50 repetitions of the simulation process. The connecting lines on top of two corresponding columns show the relative error between the averages of simulated total evacuation times represented by the two columns. The connecting lines on the bottom indicate the significant difference between the two sets of data. If a significant difference between the two sets of data were observed (for $p \leq 0.05$ scenarios), this is marked with *, else (for $p \geq 0.05$ scenarios) marked with **.

As can be seen in Fig. 4, large stair inclination scenarios lead to longer total evacuation time. Among all these particular physical setups, one concluding observation is that the effect of stair slopes on pedestrian evacuation time is basically consistent both for ascending and descending movement. For example, when the slope of the stairs changes from $20^{\circ}$ to $25^{\circ}$, the TET increases about $5.8 \%$. With the increases of $\theta$, about $12.8 \%$ and $19.3 \%$ longer TET were found. Finally, after increasing the slope to $40^{\circ}$, there were $28.4 \%$ extend in terms of the simulated TET.

Then, we investigated different stair configurations with the same stair slopes and different tread depth sizes. As presented in Fig. 5, one clear observation based on these plots is that the impact of the tread depth size differs from $3 \%$ to $27.9 \%$ in terms of the total evacuation time. Scenarios with $T=0.3 \mathrm{~m}$ have the fastest predict $T E T$ for all the tested 
Table 1 Details of the simulation settings for each specific scenario.

\begin{tabular}{l|l|l|l|l|l|l}
\hline Scenarios & $\begin{array}{l}\text { Riser } \\
\text { height, } \\
R(\mathrm{~m})\end{array}$ & $\begin{array}{l}\text { Tread } \\
\text { depth, } \\
T(\mathrm{~m})\end{array}$ & $\begin{array}{l}\text { Slope } \\
(\theta)\end{array}$ & $\begin{array}{l}\text { Free as- } \\
\text { cending } \\
\text { speed }(\mathrm{m} / \mathrm{s})\end{array}$ & $\begin{array}{l}\text { Free de- } \\
\text { scending } \\
\text { speed }(\mathrm{m} / \mathrm{s})\end{array}$ & $\begin{array}{l}\text { Free move- } \\
\text { ment head- } \\
\text { way (m) }\end{array}$ \\
\hline S1 & 0.12 & 0.35 & 20 & 0.70 & 0.82 & 1.1 \\
S2 & 0.16 & 0.35 & 25 & 0.66 & 0.78 & 1.1 \\
S3 & 0.20 & 0.35 & 30 & 0.62 & 0.73 & 1.1 \\
S4 & 0.25 & 0.35 & 35 & 0.57 & 0.67 & 1.1 \\
S5 & 0.29 & 0.35 & 40 & 0.50 & 0.60 & 1.1 \\
S6 & 0.11 & 0.30 & 20 & 0.65 & 0.79 & 1.1 \\
S7 & 0.14 & 0.30 & 25 & 0.61 & 0.75 & 0.9 \\
S8 & 0.17 & 0.30 & 30 & 0.57 & 0.70 & 0.9 \\
S9 & 0.21 & 0.30 & 35 & 0.53 & 0.65 & 0.9 \\
S10 & 0.25 & 0.30 & 40 & 0.48 & 0.58 & 0.9 \\
S11 & 0.09 & 0.25 & 20 & 0.58 & 0.76 & 0.9 \\
S12 & 0.12 & 0.25 & 25 & 0.56 & 0.72 & 0.9 \\
S13 & 0.14 & 0.25 & 30 & 0.52 & 0.67 & 0.9 \\
S14 & 0.18 & 0.25 & 35 & 0.49 & 0.62 & 0.9 \\
S15 & 0.21 & 0.25 & 40 & 0.45 & 0.56 & 0.9 \\
\hline
\end{tabular}

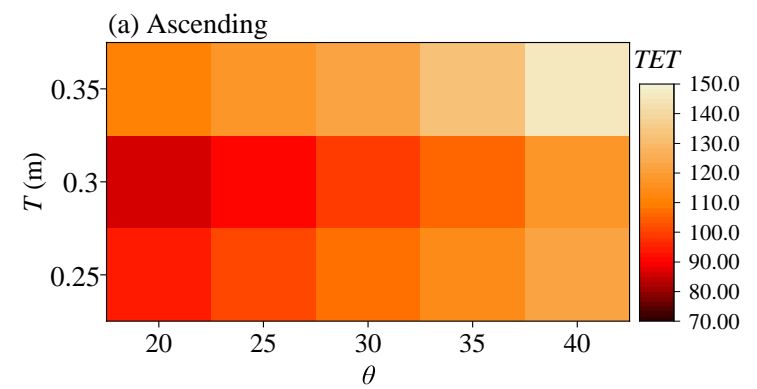

(a)

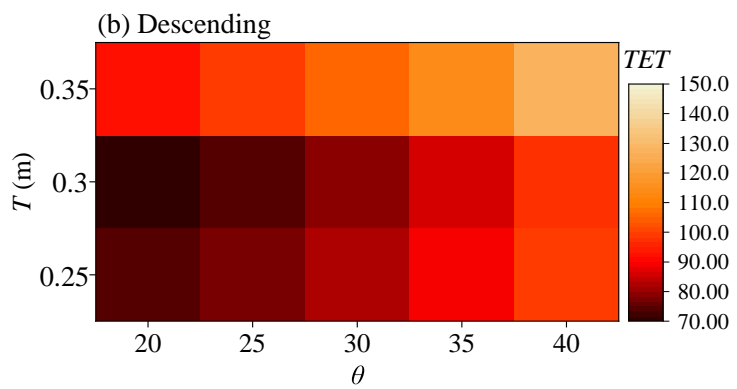

(b)

Figure 3 The color-coding magnitude of the simulated TET under various stair configurations: (a) for ascending and (b) for descending, respectively. 


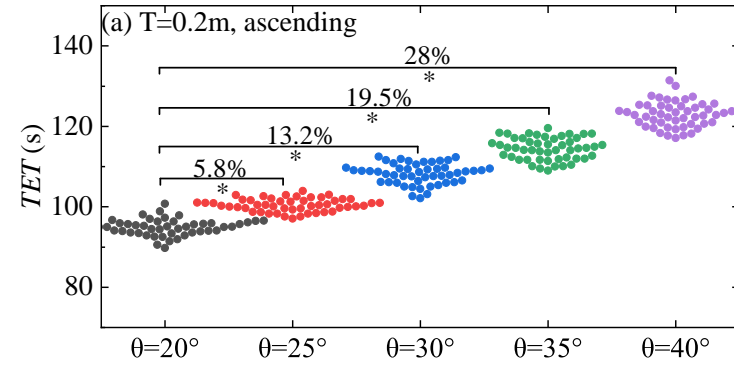

(a)

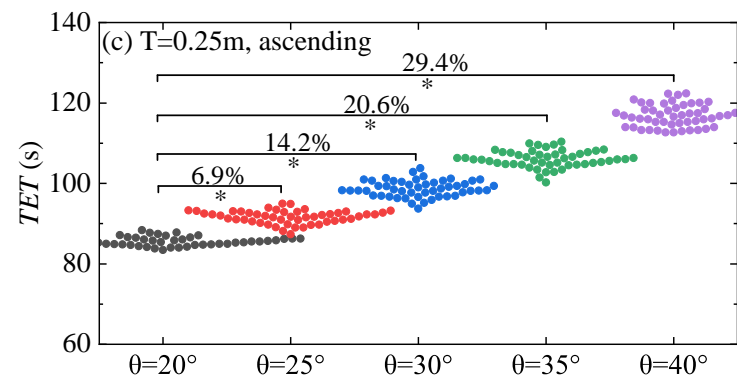

(c)

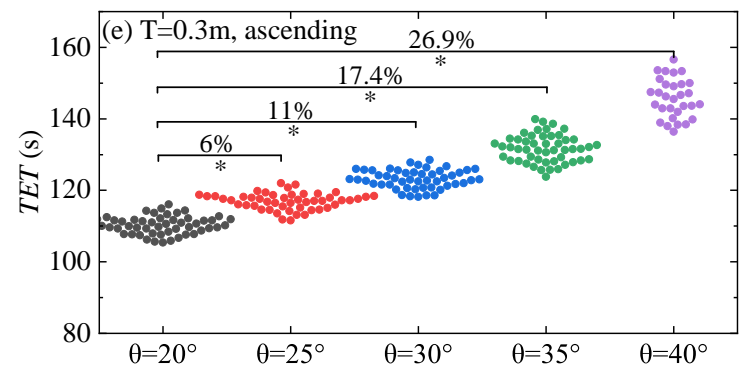

(e)

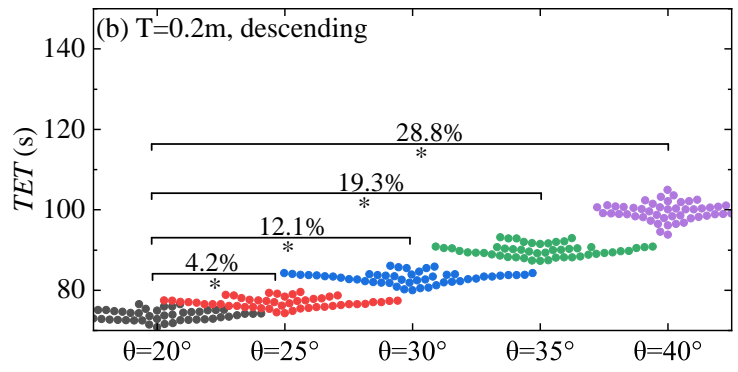

(b)

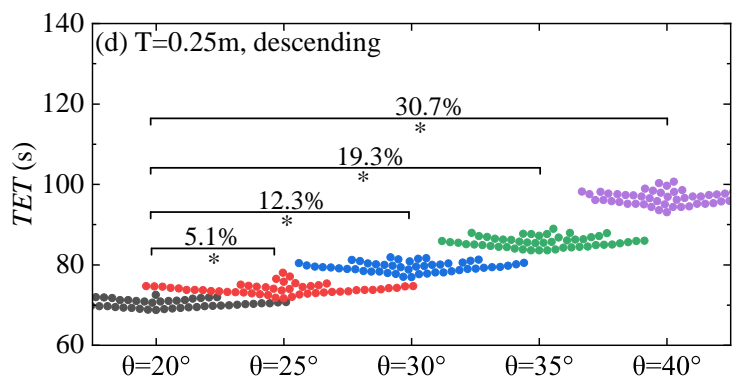

(d)

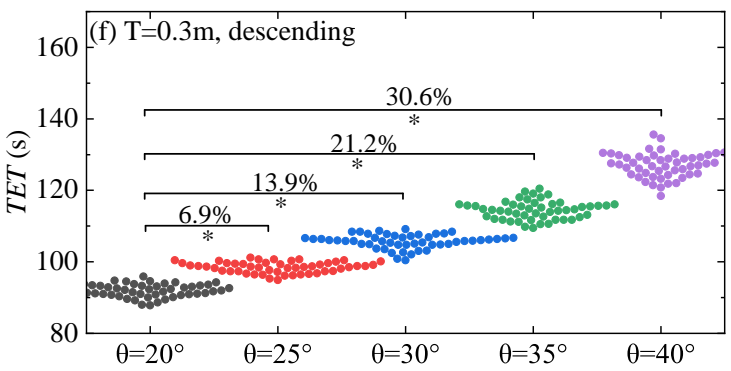

(f)

Figure 4 Comparison of the TET for different stair configurations with the same $T$ and different $\theta$.

scenarios. For $T=0.25 \mathrm{~m}$ scenarios, the $0.05 \mathrm{~m}$ tread depth changes have only minor effect on the predicted TET. And for $T=0.35 \mathrm{~m}$ series scenarios, the pedestrian flow capacity decreased by an average of $21.4 \%$.

\section{Conclusions}

In this paper, a larger number of simulation-based works have been performed to investigate how stair configuration affects pedestrian movement efficiency. The interesting conclusions are drawn based on the analysis of the total evacuation time from a series of tested scenarios. The summarized results are as follows,

(1) Our simulations quantified the effects of the stair features, including stair inclinations and tread depth, on predicted total evacuation times. Our results show that both stair 


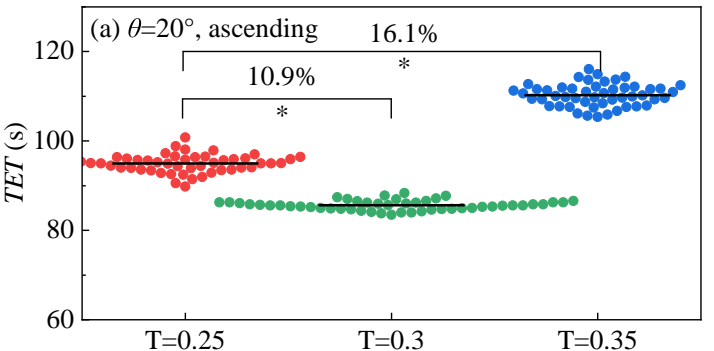

(a)

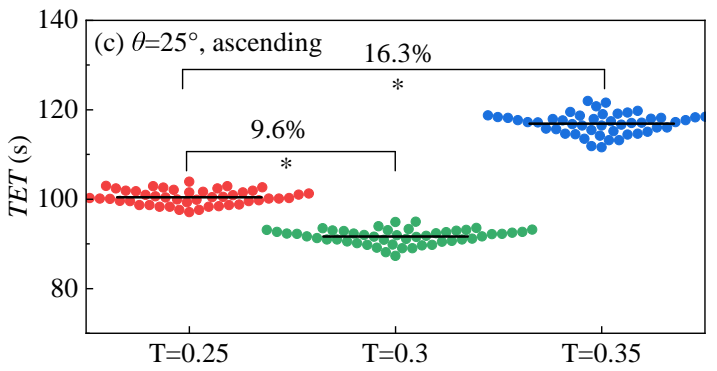

(c)

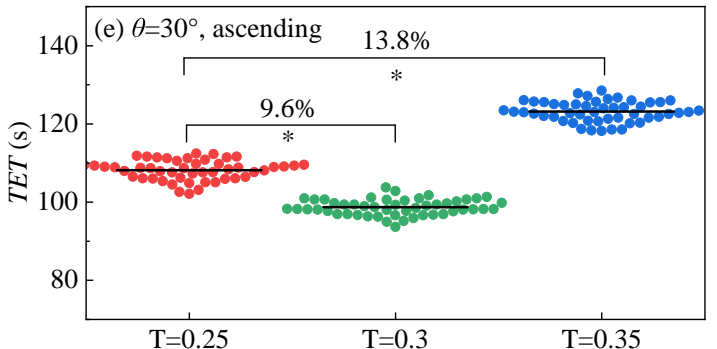

(e)

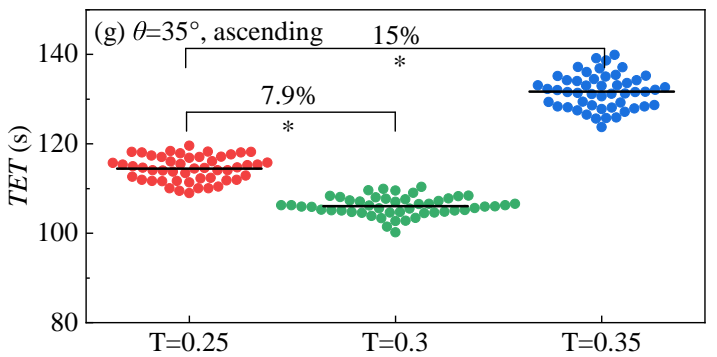

$(\mathrm{g})$

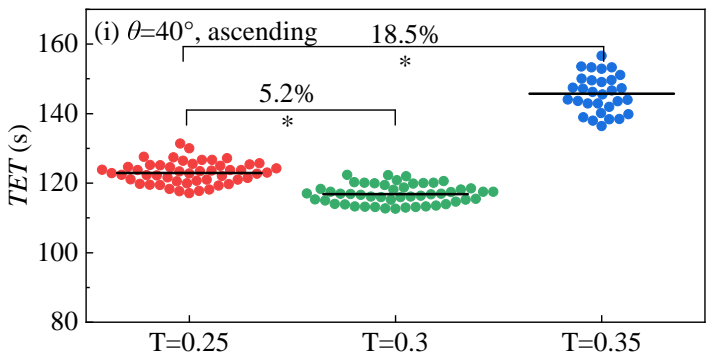

(i)

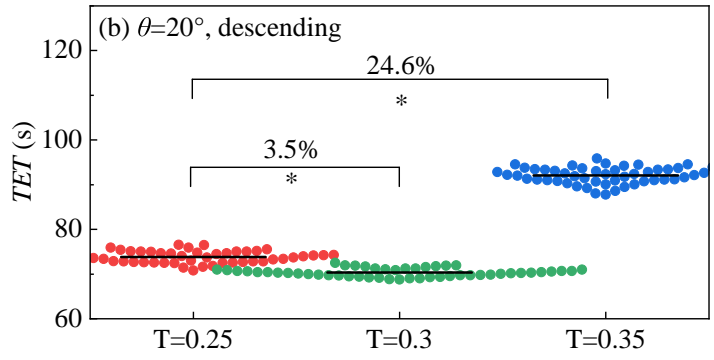

(b)

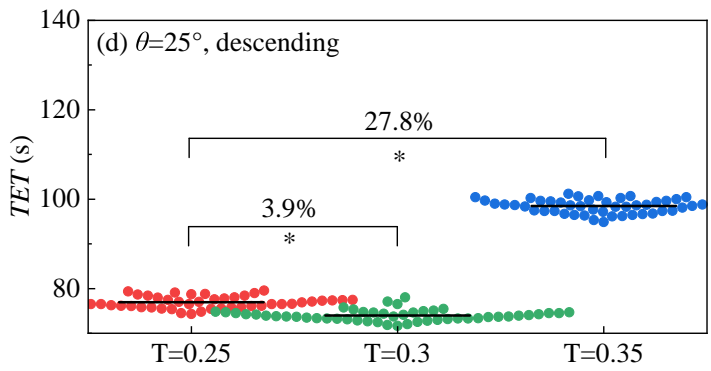

(d)

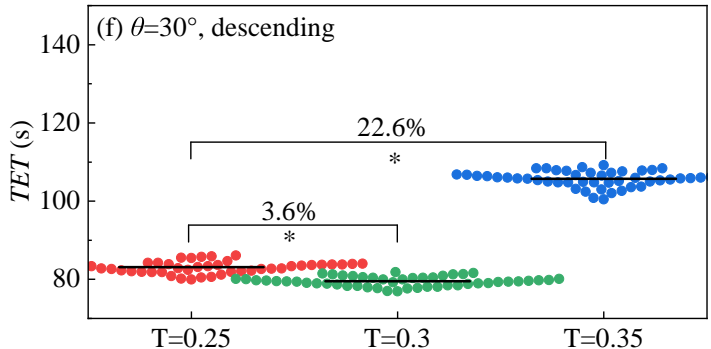

(f)

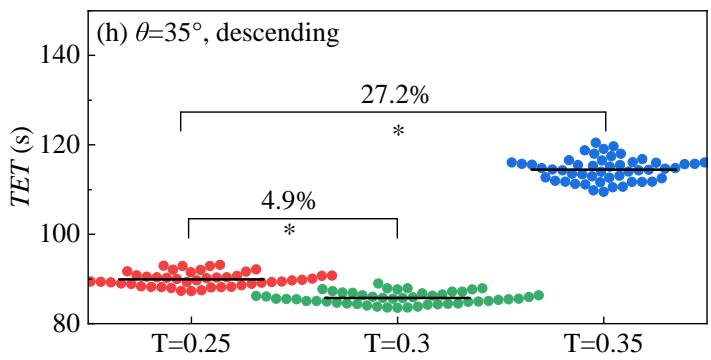

(h)

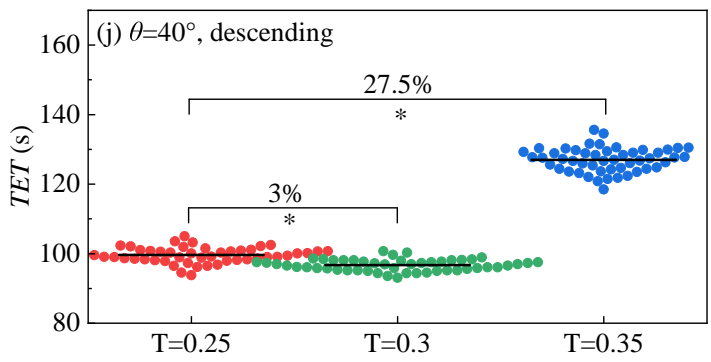

(j)

Figure 5 Comparison of the TET for different stair configurations with the same $\theta$ and different $T$. 
inclinations and tread depth are sensitive parameters which determine the total evacuation time. This highlights that in exploring the movement of pedestrians on the stairs and in designing stair geometry features, we need to consider not only the slope of the stairs, but also the depth of the steps as it relates to pedestrian motion features.

(2) We provided a deeper understanding of the relation between pedestrian flow and the slope of the stair. We found that for any tread depth, the changes of the stair slope is consistent with the change in pedestrian flow efficiency on stairs.

(3) Our simulation results suggest that the effect of the tread depth on pedestrian total evacuation time is not changed monotonously. In these particular scenarios, Scenarios with $T=0.3 \mathrm{~m}$ is the best choice. These findings further underline the importance of stair configuration in building design, as we often only consider the impact of the slope of the stairs and ignore other factors. However, it is important to note that this work is applicable for design purposes, as the work still needs further validation and research with more realistic experiments.

\section{Acknowledgement}

The work presented in this paper is supported by the National Natural Science Foundation of China (No. 71871189, 72104205), the Science and Technology Development Funds of Sichuan Province (No. 2020YFS0291), the Research Project of China Railway Eryuan Engineering Group Co.Ltd KYY2019086(19-20) and the Transport research group from the University of Melbourne.

\section{References}

[1] Chen, J., Wang, J., Wang, J., Xiang, L., Peng, L.: An experimental study of individual ascent speed on long stair. Fire Technology 53(1), 1-18 (2016)

[2] Ma, Y., Li, L., Zhang, H., Chen, T.: Experimental study on small group behavior and crowd dynamics in a tall office building evacuation. Physica A: Statistical Mechanics and its Applications 473, 488-500 (2017)

[3] Ma, J., Song, W.G., Tian, W., Lo, S.M., Liao, G.X.: Experimental study on an ultra high-rise building evacuation in china. Safety ence 50(8), 1665-1674 (2012)

[4] Sano, T., Ronchi, E., Minegishi, Y., Nilsson, D.: Modelling pedestrian merging in stair evacuation in multi-purpose buildings. Simulation Modelling Practice and Theory p. S1569190X18300522 (2018)

[5] Chen, J., Ma, J., Lo, S.M.: Geometric constraint based pedestrian movement model on stairways. Physica A: Statistical Mechanics and its Applications 505, 1212-1230 (2018) 
[6] Xuan, X., Song, W.: Staircase evacuation modeling and its comparison with an egress drill. Building \& Environment 44(5), 1039-1046 (2009)

[7] Yang, L., Ping, R., Zhu, K., Liu, S., Xin, Z.: Observation study of pedestrian flow on staircases with different dimensions under normal and emergency conditions. Safety Science 50(5), 1173-1179 (2012)

[8] Liu, R., Fu, Z., Schadschneider, A., Wen, Q., Liu, S.: Modeling the effect of visibility on upstairs crowd evacuation by a stochastic ffca model with finer discretization. Physica A: Statistical Mechanics and its Applications 531, 121723 (2019)

[9] Oven, V.A., Cakici, N.: Modelling the evacuation of a high-rise office building in istanbul. Fire Safety Journal 44(1), 1-15 (2009)

[10] Lian, L., Mai, X., Song, W., Richard, Y., Rui, Y., Jin, S.: Pedestrian merging behavior analysis: An experimental study. Fire Safety Journal 91(jul.), 918-925 (2017)

[11] Chen, S., Fu, L., Fang, J., Yang, P.: The effect of obstacle layouts on pedestrian flow in corridors: An experimental study. Physica A: Statistical Mechanics and its Applications 534, 122333 (2019)

[12] Wang, J., Ma, J., Lin, P., Sarvi, M., Li, R.: Pedestrian single file movement on stairway: Investigating the impact of stair configuration on pedestrian ascent and descent fundamental diagram. Safety Science 143, 105409 (2021)

[13] Mha, B., Ms, B.: Simulating pedestrian flow through narrow exits. Physics Letters A 383(23), 110-120 (2019)

[14] Wang, J., Sarvi, M., Ma, J., Haghani, M., Alhawsawi, A., Chen, J., Lin, P.: A modified universal pedestrian motion model revisiting pedestrian simulation with bottlenecks. Building Smulation 143, 105409 (2021)

[15] Haghani, M., Sarvi, M.: Simulating dynamics of adaptive exit-choice changing in crowd evacuations: Model implementation and behavioural interpretations. Transportation Research Part C: Emerging Technologies 103(JUN.), 56-82 (2019)

[16] Jianyu, W., Jian, M., Peng, L., Juan, C., Zhijian, F., Tao, L., Sarvi, M.: Experimental study of architectural adjustments on pedestrian flow features at bottlenecks. Journal of Statistical Mechanics: Theory and Experiment 2019(8), 083402 (2019)

[17] Haghani, M., Majid, S.: Simulating pedestrian flow through narrow exits. Physics Letters A 383(23), 110-120 (2019)

[18] Haghani, M., Sarvi, M., Shahhoseini, Z.: When 'push' does not come to 'shove': Revisiting 'faster is slower' in collective egress of human crowds. Transportation Research Part A Policy and Practice 122, 51-69 (2019) 
[19] Liao, W., Seyfried, A., Zhang, J., Boltes, M., Zheng, X., Zhao, Y.: Experimental study on pedestrian flow through wide bottleneck. Transportation Research Procedia 2, 26-33 (2014) 